\title{
Tipificaciones y nuevos sinónimos en SaPINDACEAE relacionadas a la Flora de Argentina
}

\author{
Typifications AND NEW SynONyms In Sapindaceae RELATEd to THE \\ FLora of ARgENTINA
}

María Silvia Ferrucci ${ }^{1 *}(\mathbb{D})$

1. Instituto de Botánica del Nordeste (UNNE-CONICET), Sargento Cabral 2131, 3400 Corrientes, Argentina.

*msferrucci01@gmail.com

Citar este artículo

FERRUCCI, M. S. 2021. Tipificaciones y nuevos sinónimos en Sapindaceae relacionadas a la Flora de Argentina. Bol. Soc. Argent. Bot. 56: 389-402.

DOI: https://doi. org/10.31055/1851.2372.v56. n3.33239

Recibido: 1 Jun 2021

Aceptado: 31 Ago 2021

Publicado impreso: 30 Set 2021

Editor: Roberto Manuel Salas (iD

ISSN versión impresa 0373-580X ISSN versión on-line 1851-2372

\section{SUMMARY}

Background and aims: Sapindaceae is represented in Argentina by 15 native genera with 45 species in total. In the context of the taxonomic treatment of this family for the Flora Argentina project, many names are identified that need to be typified in order to stabilize their nomenclature, as well as the clarification of classic-type material and the proposal of new synonyms. All these names belong to genera Allophylus, Athyana, Cardiospermum, Cupania, Diatenopteryx, Diplokeleba, Dodonaea, Guindilia, Paullinia, Serjania and Thinouia.

M\&M: The nomenclatural treatment is based on the analysis of protologues, plus the study of the type-material either physical or through online images. Typification is carried out according to the International Code of Nomenclature for Algae, Fungi and Plants.

Results \& Conclusions: Lectotypes are designated for 39 taxa of Sapindaceae linked to the Flora of Argentina. The holotypes of three species are clarified. Two new synonyms are proposed.

\section{KEY WORDS}

Flora Argentina, lectotypification, nomenclature, Sapindaceae.

\section{RESUMEN}

Introducción y objetivos: Sapindaceae está representada en Argentina por 15 géneros nativos con 45 especies en total. En el contexto del tratamiento taxonómico de esta familia para el proyecto Flora Argentina, se identifican numerosos nombres que precisan ser tipificados para estabilizar su nomenclatura, así como la aclaración de material tipo clásico y la propuesta de nuevos sinónimos. Todos estos nombres pertenecen a los géneros Allophylus, Athyana, Cardiospermum, Cupania, Diatenopteryx, Diplokeleba, Dodonaea, Guindilia, Paullinia, Serjania y Thinouia.

M\&M: El tratamiento nomenclatural se basa en el análisis de los protólogos, más el estudio de material tipo ya sea físico o a través de imágenes en línea. La lectotipificación se realiza de acuerdo a las indicaciones del Código Internacional de Nomenclatura para Algas, Hongos y Plantas.

Resultados y conclusiones: Se designan lectotipos para 39 taxones de Sapindaceae relacionados a la Flora de Argentina. Se aportan aclaraciones sobre los holotipos de tres especies. Se proponen dos nuevos sinónimos.

Palabras clave

Flora Argentina, lectotipificación, nomenclatura, Sapindaceae.

\section{INTRODUCCIÓN}

En la elaboración del tratamiento de la familia Sapindaceae para el proyecto de Flora Argentina, se individualizaron nombres de especies, variedades y formas pertenecientes a los géneros Allophylus L., Athyana (Griseb.) Radlk., Cardiospermum L., Cupania L., Diatenopteryx Radlk., Diplokeleba N. E. Br., Dodonaea Mill., Guindilia Hook. \& Arn., Paullinia L., Serjania Mill. y Thinouia Triana \& Planch., que requieren tipificación o aclaraciones sobre tipos. El objetivo del presente trabajo es contribuir a aclarar la situación nomenclatural de estos nombres a fin de brindar un ejemplar permanente de referencia y proponer dos nuevos sinónimos. 


\section{Materiales y Métodos}

El tratamiento nomenclatural se basó en el análisis de los protólogos de los taxones aquí publicados, en el estudio de los especímenes tipo y en la consulta de imágenes disponibles en bases de datos en línea, entre ellas se destaca JSTOR Global Plants (https://plants.jstor.org). Se han cotejado las autorías, descripciones originales, y sinonimias, consultando las bases de datos: TROPICOS (https:// www.tropicos.org/) e IPNI (https://www.ipni.org/). Se consideraron las recomendaciones de McNeill (2014) y Prado et al. (2015). El material analizado está depositado en los siguientes herbarios A, B, BA, BAF, BM, BR, C, CORD, E, F, FI, FR, G, GH, GOET, HAL, K, L, LE, LY, M, MICH, MO, MPU, NY, P, PR, R, S, TO, UC, US, W, WU y Z (acrónimos de acuerdo a Thiers, 2021). Fue de gran ayuda la información disponible en Stafleu \& Cowan (1976-1988) para consultar referencias de material tipo.

La lectotipificación se realizó de acuerdo a las indicaciones del Código Internacional de Nomenclatura para Algas, Hongos y Plantas (Turland et al., 2018), en adelante CIN. Se indican entre corchetes datos que no figuran en las etiquetas derivadas del análisis del protólogo o datos de localidad correctos y entre comillas la información del protólogo cuando es requerida. Los números que figuran a continuación de los acrónimos corresponden al código de barras, se indica con un signo de exclamación al ejemplar de herbario analizado por el autor y al ejemplar consultado en línea se lo reconoce con la palabra foto. En las citas del material depositado en el herbario BA la cifra que le sucede corresponde al número de herbario.

\section{Resultados y Discusión}

Se designan lectotipos para 23 especies, tres variedades y 13 formas; se discute la elección de los mismos. Se aportan aclaraciones sobre los holotipos de tres taxones de colecciones clásicas. Se proponen dos nuevos sinónimos. Los nombres tratados se colocan bajo los respectivos nombres actualmente aceptados que se presentan en orden alfabético (Ferrucci, 1999, 2008; Acevedo-Rodríguez et al., 2017).
Allophylus edulis (A. St.-Hil., A. Juss. \& Cambess.) Hieron. ex Niederl., Bol. Mens. Mus. Prod. Argent. 3(29): 180. 1890. $\equiv$ Schmidelia edulis A. St.-Hil., A. Juss. \& Cambess., Pl. Usuel. Bras. 1: t. 67. 1828. Tipo: Brésil [Brasil]. Province de Saint-Paul [Estado São Paulo]. Voyage d'Auguste de Saint - Hilaire, de 1816 à 1821, ad ripas amnis Rio de Tibaica, Fl X, A. de Saint-Hilaire, Catal. C ${ }^{1}$, A. de Saint-Hilaire 1063 (Lectotipo P 02297109 [foto!], designado por P. Acevedo-Rodríguez, 127. Sapindaceae, en Mota de Oliveira, S. (ed.), Flora of the Guianas. Series A: Phanerogams Fascicle 29. Royal Botanical Garden, Kew: 10. 2012; isolectotipos F 0071654 [foto! fragmento ex P], MPU 012400 [foto!], MPU 012401 [foto! 2 cartulinas], P 02297108 [foto!], P 02297110 [foto!].

$=$ Allophylus edulis Niederl. var. gracilis Radlk., en Martius, Fl. Bras. 13(3): 485.1900. Tipo: [Brasil. Minas Gerais.] Prov. Minas Geraes, Caldas, 25-IX-1875 (fl), 10-XI-1875 (fr), C. W. Hjalmar Mosén 4035 (Lectotipo aquí designado S 11-31330 [foto!]; isolectotipos S 11-31322 [foto!], S 11-31323 [foto!], S 11-31325 [foto!]).

Acevedo-Rodríguez (2012) designó como lectotipo de Schmidelia edulis una colección de St. Hilaire depositada en P, e indicó que el lectotipo se trataba de un ejemplar en flor con dos labelos de Saint Hilaire, para distinguirlo de otros dos materiales originales también depositados en $\mathrm{P}$ (isolectotipos). En el presente tratamiento se señalan los códigos de barras de los respectivos ejemplares de $\mathrm{P}$, además se encontraron tres isolectotipos depositados en F y MPU.

En el protólogo de Allophylus edulis var. gracilis, Radlkofer (1900) enumeró numerosos sintipos. Se seleccionó la colección de Mosén debido a que consta de cuatro ejemplares, dos en fruto y dos en flor, muy bien conservados, todos depositados en S (S 11-31330 fr, S 11-31322 fr; S 11-31323 fl, S 11-31325fl); entre ellos se designó al ejemplar Mosén S 11-31330 como lectotipo, pues se trata de una rama fructífera con abundante material. Acevedo-Rodríguez (2012: 10), cometió un error al tratar este taxón, pues repitió la cita incluida en la tipificación de Schmidelia edulis "Type: Brazil, São Paulo, A. de St. Hilaire 1063 (lectotype P!; isolectotypes P-2!, here designated)". 
Allophylus guaraniticus (A. St.-Hil.) Radlk., Sitzungsber. Math.-Phys. Cl. Königl. Bayer. Akad. Wiss. München 20: 312. 1890. $\equiv$ Schmidelia guaranitica A. St.-Hil., Bull. Sci. Soc. Philom. Paris 1823: 133. 1823. Tipo: Brésil [Brasil]. Rio Grande do Sul. Missoes, prope vicos St. Miguel et S. Anjo, A. de Saint-Hilaire 2744 (Lectotipo aquí designado P 02297117 [foto!]; isolectotipos MPU 012669 [foto! 2 cartulinas], P 02297116 [foto!], P 02297118 [foto!]).

Saint-Hilaire (1823) en el protólogo de Schmidelia guaranitica no menciona ninguna colección, recién lo hace Cambessèdes en $\mathrm{Fl}$. Bras. Merid. (1828), donde menciona "Nascitur in sylvis provinciae dictae Missoes, presentìm prope vicos S. Miguel et S. Anjo. Floret Martio". De los tres ejemplares que se consideran material original coleccionados por Saint-Hilaire 2744 depositados en $\mathrm{P}$, se eligió como lectotipo el ejemplar que presenta una rama florífera abundante muy bien conservada y los datos de colección manuscritos coinciden con el protólogo.

Allophylus pauciflorus Radlk., Meded. RijksHerb. 19: 61. 1913. Tipo: [Bolivia. Tarija]. Strauch im Wald bei Villa Montes (R. Pilcomayo), 460 m, XI-1910, Th. Herzog 1109 (Lectotipo aquí designado L 0013312 [foto!]; isolectotipos F $\mathrm{N}-006012$ [foto!], M 0212794! 3 cartulinas, M 0212795!, S S-R-11036! 2 cartulinas, Z 000064409 [foto!]).

Radlkofer (1913) en el protólogo de Allophylus pauciflorus menciona un único ejemplar, sin indicar el herbario en el cual está depositado por lo cual entre los cinco isosintipos disponibles se selecciona como lectotipo el único que está determinado de puño y letra por Radlkofer, además presenta la etiqueta original de colección, encabezada por un título impreso "Plantae in itinere secundo per Boliviam lectae". El ejemplar consta de una rama florífera bien conservada. Los restantes duplicados revisados no cuentan con datos de colección original, pero todos fueron revisados por Radlkofer con posterioridad a la publicación de la especie, todos tienen el mismo número y el autor adhiere al ejemplar la descripción impresa de la especie, parte en la etiqueta y completa en otra cartulina. Además, se dispone del fototipo F N-006012, del material que está depositado en $\mathrm{M}$.

Athyana weinmanniifolia (Griseb.) Radlk., en Durand, Index Gen. Phan. 73. 1888. 三 Thouinia weinmanniifolia Griseb., Abh. Königl. Ges. Wiss. Göttingen 24: 81. 1879. Tipo: Argentina. Jujuy. San Lorenzo, Baum, 4-XI-1873, G. H. E. P. G. Lorentz \& W. Hieronymus 205 (Lectotipo aquí designado G 00002049 [foto! 2 cartulinas]; isolectotipos CORD 00004955!, CORD 00004956!, F 0071831 [foto!], F N-005643 [foto!], K 000542540!, K 000542541!, S 11-24643 [foto! 2 cartulinas]).

= Athyana weinmanniifolia (Griseb.) Radlk. fo. tomentella Radlk., Pflanzenr. IV fam. 165 (Heft 98b): 431. 1932. Tipo: Argentina. Jujuy. Quinta pr. Laguna de la Brea, Exped. Suec. in reg. Chaco-Andinis, 15-VI-1901, K. R. E. Fries 186 (Lectotipo aquí designado S 11-24645 [foto!]).

Grisebach (1879) menciona en el protólogo de Thouinia weinmanniifolia el nombre vulgar y datos de localidad. Este botánico procesó las colecciones de plantas argentinas realizada por los profesores Lorentz y Hieronymus quienes trabajaron en Córdoba (Stafleu \& Cowan, 19761988). De los siete isosintipos localizados, todos ellos ligados al protólogo por los datos de colección, se designa como lectotipo al ejemplar depositado en G (G 00002049) debido a que se encuentra bien conservado, consta de dos cartulinas, una con ramas floríferas y otra rama con frutos maduros, todas desprovistas de hojas, y la segunda cartulina presenta una rama estéril con hojas y un sobre con fragmentos de hojas y mericarpos maduros. También se cuenta con un fototipo F N-005643, del material que estuviera depositado en $\mathrm{B}$.

De los dos sintipos mencionados por Radlkofer (1932) en el protólogo de Athyana weinmanniifolia fo. tomentella, se localizó únicamente el espécimen Fries 186, depositado en S (S 11-24645), el mismo consta de dos ramas estériles muy bien conservadas. El ejemplar fue identificado por Radlkofer a nivel específico en mayo de 1903.

Cardiospermum corindum L., Sp. Pl. ed. 2: 526. 1762. Tipo: Jamaica. Houston, s.n. (Neotipo BM, designado por P. W. Leenhouts en Adema, F. et al. (eds.), Fl. Malesiana, ser. 1, 11: 484. 1994).

= Cardiospermum loxense Kunth, Nov. Gen. Sp. 
(quarto ed.) 5: 102. 1821. Cardiospermum corindum L. fo. loxense (Kunth) Radlk., en Martius, Fl. Bras. 13(3): 447. 1897. Cardiospermum corindum L. var. loxense (Kunth) F. A. Barkley, Lilloa 28: 152. 1957. Tipo: [Perú]. "Crescit prope Loxa Peruvianorum, ad vias, alt. 1060 hex. Floret Augusto " F. W. H. A. Humboldt \& A. J. A. Bonpland 3399 (Lectotipo aquí designado P 00679940!; isolectotipos B B-W 07731010!, B B-W 07734-010!, P 00136898!).

= Cardiospermum parviflorum Cambess., en A. St.-Hil., A. Juss. \& Cambess., Fl. Bras. Merid. (quarto ed.) 1(9): 351.1825 [1828]. Cardiospermum corindum L. fo. parviflorum (Cambess.) Radlk., en Martius, Fl. Bras. 13(3): 447-448. 1897. Syn. nov. Cardiospermum corindum L. var. parviflorum (Cambess.) Ferrucci, en Zuloaga, F. O., O. Morrone \& M. J. Belgrano, Monogr. Syst. Bot. Missouri Bot. Gard. St. Louis 2: 2940. 2008. Nom. inval. Tipo: Brésil [Brasil]. Province [Estado] de Rio de Janeiro. Voyage d'Auguste de Saint-Hilaire, de 1816 à 1821, Aldea de S. Pedro, fl. IX, 12 VIII-XI 1818, Cat. B $74{ }^{\mathrm{s}}$ A. de St.-Hilaire s.n. (Lectotipo aquí designado P 02297034!; isolectotipos G!, MPU 010899 [foto!], P 02297035!).

= Cardiospermum halicacabum L. var. angustisectum Griseb., Abh. Königl. Ges. Wiss. Göttingen (Symb. Fl. Argent.) 24: 78. 1879. Cardiospermum corindum L. fo. angustisectum (Griseb.) Radlk., en Martius, Fl. Bras. 13(3): 448. 1897. Cardiospermum corindum L. var. angustisectum (Griseb.) F. A. Barkley, Lilloa 28: 152. 1957. Tipo: Argentina. Córdoba. Quilino, 8-XI1872, P. G. Lorentz \& G. Hieronymus 513 (Lectotipo aquí designado GOET 010705!; isolectotipo CORD 00004950!).

= Cardiospermum corindum L. fo. subglabratum Radlk., en Martius, Fl. Bras. 13(3): 447. 1897. Cardiospermum corindum L. var. subglabratum (Radlk.) F. A. Barkley, Lilloa 28: 152. 1957. Tipo: Martinique [Martinica]. Fl. Martin. № 105, 1822, [F. Kohaut] F. W. Sieber s.n. (Lectotipo aquí designado BR 0000013205768!; isolectotipos BR 0000013205775!, G 00412393!, K 000370603!, L 0384763!, L 0384765!, M 0244418!, W!, NY 01309430 [foto], P 06632867!, PR 985130!, PR 985131!, PR 985132 [foto]!).

= Cardiospermum corindum L. fo. elongatum Radlk., en Martius, Fl. Bras. 13(3): 448. 1897. Cardiospermum corindum L. var. elongatum (Radlk.) F. A. Barkley, Lilloa 28: 152. 1957. Tipo:
[Argentina]. Salta. Rio Juramento, II-1873, P. G. Lorentz \& G. Hieronymus 298 (Lectotipo aquí designado GOET 010706! 2 cartulinas; isolectotipo US 00757927!).

Kunth (1821) cita en el protólogo de Cardiospermum loxense la localidad de Perú donde fue coleccionado el material tipo "Crescit prope Loxa Peruvianorum", de los cuatro duplicados encontrados, el espécimen P 00679940 se elige como lectotipo, está identificado de puño y letra por Kunth. Mientras que el espécimen P 00136898 es un isolectotipo, material que fue donado al herbario de $\mathrm{P}$ por Bonpland con posterioridad a la publicación de la obra en la cual Kunth describió la especie, pero es parte de la colección original porque posee los datos de colectores y número. En relación a los dos especímenes depositados en B no hay indicios de que hayan sido consultados por Kunth, pero también son parte de la misma colección porque ambos tienen datos de colectores y uno de ellos B-W-07731-010 además tiene el número.

Cambessèdes (1828) en el protólogo de Cardiospermum parviflorum indica con precisión el lugar de colección "Lecta prope pagum vulgò Aldea de S. Pedro, in provinciâ Rio de Janeiro". Se ubicaron cuatro duplicados de esta colección realizada por St. Hilaire. Se designa como lectotipo el ejemplar más abundante depositado en $\mathrm{P}$ (P 02297034), el mismo consta de dos ramas floríferas, los datos de colección se completaron con la etiqueta del espécimen (P 02297035), ejemplar más pobre. El lectotipo fue visto por Radlkofer, además tiene una etiqueta impresa con la siguiente anotación "Cardiospermum Corindum L. forma 7. parviflorum m (C. parviflorum Camb.) Determ. L. Radlkofer". El espécimen depositado en G también fue identificado por Radlkofer, consta de una pequeña rama con flores, mientra los especímenes MPU 010899 y P 02297035 no fueron examinados por Radlkofer. Radlkofer (1931-1934) reconoció para $C$. corindum 14 formas, de las cuales 11 estaban citadas para América, entre ellas C. corindum fo. parviflorum. Las diferencias para reconocerlas estaban dadas por características de indumento, de los folíolos y morfología del fruto (tamaño y forma). Después de analizar el material americano, se observó que estos caracteres no eran constantes entre las formas y se adoptó el criterio de reconocer solo el nivel específico. 


\section{S. Ferrucci - Tipificaciones y nuevos sinónimos en Sapindaceae}

Grisebach (1879) en el protólogo de Cardiospermum halicacabum var. angustisectum solo señala características de hoja y fruto para este taxón e indica con la letra $\mathrm{C}$ que ha sido coleccionada en la provincia de Córdoba; se hallaron dos duplicados (uno en GOET y otro en CORD) de una colección de Lorentz \& Hieronymus de Córdoba que concuerda con la cita del protólogo. El espécimen GOET 010705 posee una etiqueta que lee: Cardiospermum halicacabum L. var. angustisectum Gr., de puño y letra de Grisebach, por lo cual se selecciona como lectotipo.

De los tres sintipos mencionados por Radlkofer (1897) en el protólogo de Cardiospermum corindum fo. subglabratum, los cuales estaban identificados bajo Cardiospermum grandiflorum non Sw., se localizaron dos de ellos, un material de Bertero coleccionado en Puerto Rico (G 00211804) y un espécimen de Sieber colectado en Martinica. Se eligió para lectotipificar este último por tratarse de una rama florífera bien preservada, con numerosos duplicados depositados en diferentes herbarios.

Radlkofer (1897) en el protólogo de Cardiospermum corindum fo. elongatum cita tres ejemplares, Bridges s.n., Hb. Boiss.; Lorentz \& Hieronymus 298 y Lorentz \& Hieronymus 1199. Se encontraron especímenes correspondientes a los tres sintipos. Se elige como lectotipo de esta forma al espécimen Lorentz \& Hieronymus 298 depositado en GOET (GOET 010706) por presentar dos cartulinas con abundante material, con flores y frutos maduros, presenta una etiqueta con el nombre del taxón con letra de Radlkofer (IV.1897); el duplicado depositado en US (US 00757927) también es un ejemplar muy bueno con flores y frutos maduros, el mismo fue enviado por el herbario B.

Cardiospermum grandiflorum Sw., Prodr. 64. 1788. Tipo: Jamaica. Swartz s.n. (Holotipo S 111933!).

$=$ Cardiospermum velutinum Hook. \& Arn., Bot. Misc. 3: 158. 1833. Tipo: Uraguay. [Uruguay]. $S$. $d$., Tweedie s.n. (Lectotipo aquí designado K 000037453!; isolectotipos K 000037452!, K 000037454!, P 02297039!).

Swartz (1788) en el protólogo de Cardiospermum grandiflorum solo indica el país de colección del ejemplar original "Jamaica". En el herbario S donde se encuentran los ejemplares estudiados por Swartz (Stafleu \& Cowan, 1976-1988) se halla depositado un único espécimen identificado de puño y letra por Swartz, el mismo consta de una rama florífera y partes de paredes carpelares del fruto, en el sobre se observan semillas. No se han localizado duplicados en otros herbarios.

Hooker \& Arnott (1833) en el protólogo de Cardiospermum velutinum citan "Missions of Brasil, Tweedie". Se hallaron cuatro duplicados de una colección de Tweedie, tres duplicados en $\mathrm{K}$ y uno en P. Sin embargo en el espécimen colectado por Tweedie está escrito siempre Uraguay, no registrando otros datos de localidad, fecha o número. El espécimen K 000037453 es el seleccionado como lectotipo por ser el mejor ejemplar y el único que exhibe frutos maduros y semillas en el sobre; consta de dos ramas floríferas, una de ellas además con frutos. El mismo está identificado de puño y letra por los autores. De los otros dos ejemplares depositados en K, el K 000037454 presenta una nota ilegible.

Cupania vernalis Cambess., en A. St.-Hil., A. Juss. \& Cambess., Fl. Bras. Merid. (quarto ed.) 1(10): 387.1825 [1828]. Tipo: Brésil [Brasil]. Province [Provincia] de Rio Grande do Sul. Voyage d'Auguste de Saint-Hilaire, de 1816 à 1821, Estancia de José Bernardez, fl. IX, s. l. (Lectotipo aquí designado P 02297418 [foto!]; isolectotipos MPU 010933 [foto!], P 00481903 [foto!], P 02297417 [foto!]).

= Cupania uraguensis Hook. \& Arn., en Bot. Misc. 3: 159. 1833. Tipo: Uraguay [Uruguay]. $S$. d., J. Tweedie s.n. (Lectotipo aquí designado $\mathrm{E}$ 00502906 [foto!]).

Cambessèdes (1828) en el prótologo de Cupania vernalis indica el lugar de colección sin referir un espécimen en particular. Se encontraron cuatro especímenes que se corresponden con los datos de colección. El protólogo hace referencia a caracteres vegetativos y solo a caracteres reproductivos florales. Se elige como lectotipo el ejemplar depositado en P (P 02297418), consta de una rama florífera, la misma está muy bien conservada y tiene la etiqueta con los datos de colección citados en el protólogo. Este ejemplar posee además una etiqueta impresa con esta leyenda "Cupania vernalis Camb. f. 1 genuina m. Determ. L. Ralkofer". 
Hooker \& Arnott (1833) en el protólogo de Cupania uraguensis describen un material florífero, coleccionado por Tweedie en Uruguay. Se localizó un ejemplar que coincide con el protólogo en $\mathrm{E}$, (E 00502906) el cual se elige como lectotipo, está determinado de puño y letra con las iniciales $\mathrm{H} \&$ A, y está escrito en la etiqueta "Uraguay, Tweedie".

Diatenopteryx sorbifolia Radlk., Sitzungsber. Math.-Phys. Cl. Königl. Bayer. Akad. Wiss. München 8: 285. 1878. Tipo: [Brasil]. Prov. Minarum [Minas Geraes]. Serra de Caldas, 1861, A. F. Regnell I 118** (Lectotipo aquí designado BR 0000013204426 [foto!]).

= Thouinia ornifolia Griseb., Abh. Königl. Ges. Wiss. Göttingen 24: 82. 1879. Tipo: Argentina. [Salta]. Orán, nom. vern. Suiquillo, IX-X-1873, P. G. Lorentz \& G. Hieronymus 42 (Lectotipo aquí designado GOET 010715!; isolectotipos BAF 00000239!, BAF 00000240!, BR 0000013205942 [foto!], CORD 00004958!, CORD 00004959!, F N-23612 [foto], FR 0036052 [foto!], GOET 010716!, K 000542549!, M 0212714 [foto!], P 06695838 [foto!], S 11-24649 [foto!], UC 870382 [foto!]).

Entre los sintipos Sello n. 2214, Regnell I $n .118^{* *}$ y Regnell III n.1564 mencionados por Radlkofer (1878) en el protólogo de Diatenopteryx sorbifolia, el espécimen Regnell I 118** depositado en el herbario BR (BR 0000013204426), se elige como lectotipo por tratarse de una rama florífera muy abundante y bien conservada, identificada de puño y letra por Radlkofer.

En el protólogo de Thouinia ornifolia, Grisebach (1879) refiere dos colecciones, una procedente de Jujuy, San Lorenzo y otra de Salta, Orán, ambas coleccionadas por Lorentz \& Hieronymus, esta última está muy bien representada en los herbarios. El ejemplar aquí seleccionado como lectotipo, GOET 010715, tiene los datos de etiqueta que coinciden con el protólogo, consta de dos ramas floríferas y un sobre con folíolos, restos de inflorescencias y al menos un mericarpo; además tiene detalles de la flor dibujados a lápiz, se trata de dibujos muy básicos de las piezas florales. El ejemplar depositado en P (P 06695838) coincide en todos sus datos con el protólogo solo falta el número. Además, se dispone del fototipo $\mathrm{F}$ $\mathrm{N}-006012$, espécimen fotografiado en G.
Diplokeleba floribunda N. E. Br., en Kerr, Trans. \& Proc. Bot. Soc. Edinburgh 20(1): 50. 1894. Tipo: [Paraguay]. Fortin Page, $24^{\circ} 50^{\prime} \mathrm{S}$. latitude, Rio Pilcomayo Expedition 1890-91, J. G. Kerr 85 (Holotipo K 000586263!; isotipo NY 00337828 [foto! fragmento ex K]).

Brown (en Kerr 1894) ), asistente en el herbario de Kew, en el protólogo de Diplokeleba floribunda cita una única colección, Kerr 85. Este material se encuentra depositado en $\mathrm{K}$ (K 000586263), presenta dos ramas floríferas muy bien conservadas, y es el holotipo. El material de NY 00337828 es un fragmento de material florífero procedente de K (escrito en una etiqueta), y se trata por lo tanto de un isotipo. Se destaca que en el isotipo se aprecia un material adicional en fruto que además está ilustrado a lápiz y en un sobre se observan partes de un fruto y una semilla, este material corresponde a una colección posterior realizada por T. Rojas, Herb. Hassler, lect. $\mathrm{N}^{\circ} 360$ depositado en $\mathrm{G}$, este complemento lo realizó B. P. G. Hochreutiner en 1907.

Dodonaea viscosa Jacq., Enum. Syst. Pl.: 19, f. 5. 1760. Tipo: Jamaica. H. Sloane s.n., fr. (Lectotipo BM 000589176 [foto], designado por J. G. West, Brunonia 7(1): 34. 1984).

= Dodonaea burmanniana DC., Mém. Soc. Phys. Genève 1: 447. 1822. Dodonaea viscosa Jacq. fo. burmanniana (DC.) Radlk., Fl. Bras. 13(3): 646. 1900. Dodonaea viscosa Jacq. ssp. burmanniana (DC.) J. G. West, Brunonia 7(1): 37. 1984. Tipo: India. Bengal, 1821, L. T. Leschenault de la Tour s.n. (Lectotipo aquí designado G 00214325 [foto!]).

De Candolle (1822) en el protólogo de Dodonaea burmanniana señala que se basa en la figura 28 de Burman en La Flora de Ceylon. West (1984) menciona que el herbario de De Candolle (G-DC), que constituye la base de su tratamiento del género (De Candolle 1824), cuenta con tres especímenes que serían material original coleccionados, uno en Ceylan (actualmente Sri Lanka), otro en Bengal y el tercero en Timor. Sin embargo no elige un lectotipo, razón por la cual después de haber localizado estos tres sintipos, se propone designar como lectotipo al espécimen de Bengal, G 00214325, por estar representado 
por una rama con flores y frutos inmaduros muy bien conservada. También se han localizado dos posibles duplicados del espécimen G 00214324 , coleccionado en Timor, uno de ellos depositado en MPU (MPU 1213115) y el otro depositado en $\mathrm{P}$ (P 00337524), en ambos está manuscrito Dodonaea Burmanniana DC., en la etiqueta está impreso Timor; sin embargo no los he considerado como sintipos por contar con escasa información. Leschenault de la Tour fue un botánico francés contemporáneo de De Candolle, en el herbario $\mathrm{G}$ se encuentran numerosos duplicados de sus colecciones correspondientes a diversas familias que han servido de base para la descripción de nuevas especies por parte de De Candolle y de otros botánicos.

Guindilia trinervis Gillies ex Hook. \& Arn., en W. J. Hooker, Bot. Misc. 3: 170. 1833. Tipo: [Chile]. Cordilleras of Chili, Cuming 290 (Lectotipo aquí designado K 000542546!; isolectotipos $\mathrm{E}$ 00296713 [foto!], E 00296715 [foto!]).

= Valenzuelia trinervis Bertero ex Cambess., Nouv. Ann. Mus. Hist. Nat. 3: 236 t. 14. 1834. Tipo: Chili. [Chile]. [Rancagua] Mont. la Leona, $\mathrm{O}^{\text {br }}$. 1828, D. Bertero 738 (Lectotipo aquí designado P 06695869 [foto!]; isolectotipos BM 013842686!, BR 0000013205522 [foto!], CORD 00003242! fragmento ex herb. Colla, F N-005644 [foto!], GH 00051009 [foto!], GH 00051010 [foto!], L 03113220 [foto!], M 0212715 [foto!], MPU 010896 [foto!], MPU 010897 [foto!], P 06695867 [foto!], P 06695868 [foto!], P 06695870 [foto!], TO [foto! cartulina 1374, Herbarium Colla).

Hooker \& Arnott (1833) en el protólogo de Guindilia trinervis citan dos sintipos, uno de ellos coleccionado por el autor de la descripción de la especie, Gillies 62 y el otro coleccionado por Cuming 290. Están disponibles las imágenes de ambas colecciones depositadas en los herbarios E y K. En general la colección Cuming 290 está mejor representada, en lo que se refiere a los especímenes con flores y frutos. El duplicado que muestra la mejor calidad de preservación y exhibe frutos maduros (K 000542546) es elegido aquí como lectotipo del nombre. Se destaca que el autor describió la especie sobre caracteres vegetativos y carpológicos, de la flor solo hay restos. En la cartulina donde está el ejemplar aquí designado como lectotipo, se encuentran tres ejemplares más, cada uno con sus datos y código de barra, separados con trazos en lápiz. Uno de estos tres es el sintipo Gillies 62 que está representado por una rama estéril. Los otros dos ejemplares, evidentemente son colecciones posteriores y corresponden a ramas floríferas.

En el protólogo de Valenzuelia trinervis, Cambessèdes (1834) cita solo la localidad de la colección "In sylvis montis La Leona". Entre los catorce duplicados detectados, cuyos datos de colección concuerdan con el protólogo, se selecciona como lectotipo el espécimen depositado en P (P 06695869) por contar con una etiqueta manuscrita con los siguientes datos (Valenzuelia trinervis Bertero, genus novum, Mont. La Leona, D. Bertero $8^{\text {bre }} 1828$ ), es un excelente ejemplar que consta de dos ramas con flores y frutos inmaduros, el mismo corresponde al Ex herb. Di Guillemin 1842. Se destaca que el espécimen P 06695867 consta de dos ramas con flores y frutos inmaduros. Este ejemplar presenta una descripción de este nuevo género extensa y manuscrita, la cual finaliza con los datos de la etiqueta, está escrita en dos trozos de papel y en el segundo dice en el encabezado en distinta tinta (rojiza) Herbarium Steudel y al finalizar agrega Bertero. En una etiqueta impresa con el dato Herb. Mus. Paris está escrito con lápiz Valenzuelia trinervis Bert. En comunicación personal con la Dra. Laura Guglielmone, Curadora de Fanerógams del herbario TO, corroboré que las muestras recogidas en Chile por Bertero fueron enviadas a Turín por Delessert. Casi todas estas muestras se conservan ahora en la colección de Luigi Colla (TO) Además, se dispone del fototipo $\mathrm{F}$ $\mathrm{N}-005644$, espécimen fotografiado en B.

Paullinia pinnata L., Sp. $P l .:$ 366. 1753. Tipo: "Clematis pentaphylla, pediculis alatis, fructu racemoso, tricocco \& coccineo" en Plumier, Descr. Pl. Amér. 76, t. 91.1693 (Lectotipo designado por F. G. Davies en Kew Bull. 32(2): 430. 1978).

$=$ Paullinia angusta $\mathrm{N}$. E. Br., en Kerr, Trans. \& Proc. Bot. Soc. Edinburgh 20(1): 49. 1894. Tipo: Paraguay. [Depto. Villa Hayes] Villa Occidental. [Villa Hayes], tiges grimpantes, fleurs blanches, fruits rouges, 3-V-1874, B. Balansa 2479 (Lectotipo aquí designado K 000072614 [foto!]). 
De los cuatro sintipos mencionados por Brown (en Kerr 1894) en el protólogo de Paullinia angusta se localizaron tres depositados en $\mathrm{K}(\mathrm{K}$ 000072615; K 000544373; K 000072614), el último de los citados fue elegido como lectotipo por tratarse de un ejemplar muy bien conservado con datos completos de etiqueta, está representado por una rama con frutos maduros y complementa con una hoja. Está determinado de puño y letra por Brown y hace referencia a los datos de publicación, incluyendo la palabra "Type".

Serjania caracasana (Jacq.) Willd., Sp. Pl. 2: 465. 1799. $\equiv$ Paullinia caracasana Jacq., Pl. Hort. Schoenbr. 1: 52, t. 99. 1797. Tipo: [Venezuela]. Jacquin's Hort. Schoenb.Tabula 99. 1797.

= Serjania nitidula Benth., Hooker's J. Bot. Kew Gard. Misc. 3: 191. 1851. Serjania caracasana fo. nitidula (Benth.) Radlk., Monogr. Serjania 148. 1875. Tipo: [Brasil]. Prov. [Estado] Parà. In vicinibus Santarem, VIII-1850, R. Spruce 1013 (Lectotipo aquí designado K 000037161!; isolectotipos BM 013842510 [foto!], F 0071799 [foto!], G 00008060 [foto!], G 00008133 [foto! 2 cartulinas], GH 00050939 [foto!]).

Serjania caracasana fo. puberula Radlk., Monogr. Serjania 148. 1875. Tipo: Bolivie [Bolivia]. Voyage de A. D'Orbigny dans 1'Amérique Méridionale, Sa. Ana de Chiquitos, 1842, A. D. D'Orbigny 679 (Lectotipo aquí designado BR 000000590532 [foto!]; isolectotipos P 05242590 [foto!], P 05242591 [foto!], P 05242593 [foto!], P 05242595 [foto!]).

Bentham (1851) en el protólogo de Serjania nitidula menciona a Santarem como la localidad de colección, este botánico trabajaba en $\mathrm{K}$ donde está su herbario y tipos (Stafleu \& Cowan, 19761988). Se encontraron duplicados de los dos sintipos depositados en K, (K 000037161 y K 000037160), sobre la base de los cuales se describió la especie. Ambos especímenes tienen como dato de localidad "Santarem" e "in vicinibus Santarem" respectivamente, fueron coleccionados en distintos meses. Se seleccionó como lectotipo el espécimen K 00003761 coleccionado en agosto de 1850 que está representado por una rama florífera, es el mejor representado en los herbarios.

Radlkofer (1875) en el protólogo de Serjania caracasana fo. puberula cita como única colección el espécimen D'Orbigny n. 679, del cual se han localizado cinco duplicados depositados en los herbarios BR y P. Entre éstos se ha seleccionado como lectotipo el espécimen depositado en BR (BR 000000590532) que consta de una rama florífera con algunos frutos inmaduros, los restantes duplicados solo presentan ramas floríferas; el mismo está identificado de puño y letra por Radlkofer.

Serjania confertiflora Radlk., Consp. Sect. Sp. Serjan.: 4. 1874. Tipo: Brasil. Rio de Janeiro, H. W. Schott 712 (Lectotipo K 000037129 !, designado por P. Acevedo-Rodríguez, Mem. New York Bot. Gard. 67: 84. 1993; isolectotipos F catál. 874637 [foto], NY 01546700!, MO 100939180 [foto], W 0058685 [foto]).

= Serjania confertiflora var. dasycephala Radlk., Mem. Torrey Bot. Club 6: 21. 1896. Tipo: Bolivia. [La Paz]. Guanai to Tipuani, IVVI-1892, M. Bang 1262a (Lectotipo aquí designado G 00008067 [foto!]; isolectotipos F $0071782 \mathrm{~F}$ [foto!], G 00008069 [foto!], GH 00216531 [foto!], K 000037343 [foto!], M 0212229 [foto!], NY 01546669 [foto!], US 00095093 [foto!], WU 0069150!]).

Serjania confertiflora Radlk. var. dasycephala fo. subincana Radlk., Repert. Spec. Nov. Regni Veg. 8: 72. 1910. Tipo: [Paraguay]. N. Paraguay. Zwischen Rio Apa und rio Aquidaban, Centurion, weiss mit orange, bis $3 \mathrm{~m}$ horhsteigend, zw. Gesträuch am Rande des Bergwaldes, 22-23 lat., XII-1908/1909, K. Fiebrig 4598 (Lectotipo aquí designado G 00008070 [foto! 2 cartulinas]; isolectotipos BM 000087737!, G 00008071 [foto! 2 cartulinas], GH 00216523 [foto!], LY 0106716 [foto!], LY 0106717 [foto!], M 0212225 [foto!]).

Radlkofer (1896) en el protólogo de Serjania confertiflora var. dasycephala cita cuatro sintipos. Las características que definen la variedad están dadas por caracteres carpológicos complementados con detalles de epidermis de los folíolos. Por esta razón se eligió el ejemplar Bang 1262a, que consta de una rama con frutos maduros, este espécimen está muy bien distribuido en los herbarios del hemisferio norte. El ejemplar depositado en $\mathrm{G}$ ( $\mathrm{G}$ 00008067) se eligió como lectotipo por ser el único entre los duplicados disponibles determinado por Radlkofer de puño y letra en 1895 . Se destaca que en los ejemplares con datos de etiqueta completos el año de colección indicado es 1892 . 


\section{S. Ferrucci - Tipificaciones y nuevos sinónimos en Sapindaceae}

Radlkofer (1910) en el protólogo de Serjania confertiflora var. dasycephala fo. subincana solo menciona el ejemplar Fiebrig 4598 depositado en el herbario Hassler. Se localizaron siete duplicados de la colección citada, de los dos guardados en el herbario G, el espécimen G 00008070 se eligió como lectotipo por estar identificado de puño y letra por Raldkofer, quien además en una nota manuscrita describe las dos formas y cita el material estudiado (Radlkofer XI.09); el espécimen consta de una buena rama con flores y frutos.

Serjania foveata Griseb., Abh. Königl. Ges. Wiss. Göttingen 19: 108-109. 1874. Tipo: Argentina. [Tucumán]. Siambon, Sierra de Tucuman, III-1872, P. G. Lorentz 288 (Lectotipo aquí designado GOET 038039 [foto!]; isolectotipos BA 50880!, CORD 00004948 [foto!], F N-005547[foto!], G 00008086 [foto! 2 cartulinas], P 05242268 [foto! fragmento ex GOET]).

Grisebach (1874) al describir Serjania foveata, cita una colección realizada en Siambón, Sierra de Tucumán. Se han localizado cinco duplicados que concuerdan con estos datos de localidad, aparte se cuenta con un fototipo F N-05547, del material que estuviera depositado en $\mathrm{B}$, este $\mathrm{N}$-material ya no existe allí, debido al bombardeo de Berlín durante la Segunda Guerra Mundial. El duplicado GOET 038039 se elige como lectotipo por constituir el material original sobre el cual Grisebach fundó la especie, tiene un labelo con dibujos muy simples de caracteres florales; fue visto por Radlkofer, con indicación de puño y letra al igual que el ejemplar depositado en $G$ ( $G$ 00008086). La diagnosis contempla solo caracteres vegetativos y florales. Destaco que el fragmento depositado en $\mathrm{P}$ (P 05242268) está en un sobre cerrado, tiene un labelo escrito a máquina donde se lo indica como "syntype". $\mathrm{El}$ isolectotipo depositado en BA no tiene indicación de mes ni número de colección pero sí de localidad y coleccionista, mientras que el isolectotipo depositado en CORD presenta los datos de etiqueta completos.

Serjania glabrata Kunth, en Humb., Bonpl. \& Kunth, Nov. Gen. Sp. 5: 110.1821. Tipo: Perú. Colazei, A. J. A Bonpland \& F. W. H. A von Humboldt 3580 (Lectotipo P 00679944, designado por A. A. Massing \& S. T. Sfoggia Miotto, Phytotaxa 428(2): 159. 2020).
= Serjania fulta Griseb., Abh. Königl. Ges. Wiss. Göttingen 19: 108. 1874. Tipo: [Argentina. Tucumán]. Bei Siambon, III-1872, P. G. Lorentz 713 (Lectotipo aquí designado GOET 010847 [foto!]; isolectotipos BA 50881!, CORD 00004947!).

= Paullinia brachystachya Griseb., Abh. Königl. Ges. Wiss. Göttingen 19: 109. 1874. Tipo: [Argentina. Tucumán]. Bei Pass Rozo al alto 2 Leguas vor Tucuman, Windendes, weinrebenartiges Gewächs, zwischen niederem Gebüsch, 22-XII-1871, P. G. Lorentz 50 (Lectotipo aquí designado GOET 010848 [foto!]; isolectotipos CORD 00004949!, GOET 010849 [foto!]).

= Serjania glabrata Kunth fo. mollior Radlk., Monogr. Serjania 169. 1875. Tipo: Brésil [Brasil]. [Estado Alagoas] Province D'Alagoas, II-IV-1838, M. Gardner 1259 (Lectotipo aquí designado G 00008108 [foto!]; isolectotipos BM 013732918, [foto!], F [foto!, V 0305348], FI 004595 [foto!], NY 1546638 [foto!], NY 1546652 [foto!], P 02296756 [foto!], P 05242337 [foto!], P 05242338!).

En el protólogo de Serjania fulta, Grisebach (1874) cita la localidad de colección, "Siambon". Se detectaron tres ejemplares que coinciden con los datos del protólogo y son material original. Entre ellos se elige al ejemplar depositado en GOET como lectotipo, debido a que el mismo estuvo a disposición del autor, presenta un dibujo elaborado a mano con anotaciones también en lápiz y una etiqueta manuscrita con el número, los datos de localidad y el nombre de la especie. Este ejemplar presenta además una etiqueta manuscrita con la identificación de Radlkofer bajo "Serjania glabrata Kunth forma 2 mollior m".

Grisebach (1874) en el protólogo de Paullinia brachystachya detalla la localidad de colección, "Rozo". El ejemplar coleccionado está depositado en GOET, donde hay dos cartulinas, una de ellas con la identificación manuscrita en tinta, además de dos anotaciones independientes de la etiqueta una con detalles básicos de flor y otra con anotaciones, ambas con lápiz. Además este ejemplar tiene una etiqueta manuscrita por Radlkofer "Serjania glabrata Kunth forma 2. mollior m." Este espécimen fue seleccionado como lectotipo, la otra cartulina tiene la etiqueta con los datos impresos. De acuerdo al Art. 8.3 según CIN (Turland et al., 2018) los ejemplares depositados en 
GOET al no presentar una etiqueta original en común se tratan como duplicados.

Radlkofer (1875) al describir S. glabrata fo. mollior cita en el protólogo siete colecciones de Martius (1), Gardner (5) y Weir (1). Como resultado del análisis de los sintipos disponibles en línea, la colección de Gardner 1259 depositada en G (G 00008108) se elige como lectotipo, la cual fue identificada por Radlkofer de puño y letra. De los tres duplicados de esta colección, depositados en P, solo el espécimen P 05242338 no está identificado de puño y letra por Radlkofer, es una rama florífera en buen estado de conservación. El ejemplar depositado en BM de acuerdo a Stafleu \& Cowan (1976-1988) correspondería a la colección original de Gardner, tiene los siguientes datos de colección escritos en tinta "Ilha de São Pedro, Rio de São Francisco, Feb. 1838”, con lápiz está escrito Serjania glabrata Kunth forma 2, Radlk.

Serjania glutinosa Radlk., Consp. Sect. Sp. Serjan. 5. 1874. Monogr. Serjania 126. 1875. Tipo: [Brasil]. Mato Grosso. Cuyabá, IV-1827, L. Riedel 938 (Lectotipo aquí designado NY 01546602 [foto!]; isolectotipos A 00050932 [foto!]; LE 00018191 [foto!], LE 00018192 [foto!], M 0212327 [foto! fragmento ex LE]).

= Serjania glutinosa fo. multiseta Radlk., Monogr. Serjania 126. 1875. Tipo: Brasil. Prov. [Est.] Minas Geraes. Caldas, IV-1846, A. F. Regnell III 342 (Lectotipo aquí designado M 0212326 [foto!]; isolectotipos BR 0000005904112 [foto!], BR 0000005904471 [foto!], BR 0000005905621 [foto!], BR 0000005904808 [foto!], C 10018699 [foto!], C10018700 [foto!], K 000037140!, S 1124338 [foto! 2 cartulinas], S 11-24339 [foto!], S 11-24340 [foto!]).

Entre los cuatro sintipos citados en el protólogo de Serjania glutinosa por Radlkofer (1875), se seleccionó como lectotipo la colección de Riedel depositada en NY (NY 01546602) por constar de una rama florífera bien representativa y conservada. El ejemplar depositado en $\mathrm{M}$ es un clastotipo del material de LE, consta de una ramita con una inflorescencia y otra con una hoja, pero del total de duplicados es el único que Radlkofer señala como visto con un signo de admiración.

Radlkofer (1875) en el protólogo de Serjania glutinosa fo. multiseta cita un único ejemplar,
Regnell III 342. Se han localizado 11 duplicados, de acuerdo con la diagnosis, datos de coleccionista y número. Éstos están distribuidos en los herbarios BR, C, K, M y S. El duplicado seleccionado como lectotipo, está identificado de puño y letra por Radlkofer, es un excelente ejemplar florífero, tiene además un fruto inmaduro pegado.

Serjania hebecarpa Benth., Hooker's J. Bot. Kew Gard. Misc. 3: 192. 1851. Tipo: Brasil. Ceará. G. Gardner 1498 (Lectotipo P 02296765 , designado por A. A. Massing \& S. T. Sfoggia Miotto, Phytotaxa 428(2): 159.2020; isolectotipos G 00008116, G 00008117, GH 00050937, K 000037249, NY 01546621, NY 01546622, NY 01546625, P 02296766, S 11-24343).

= Serjania hebecarpa fo. oxycephala Radlk., Abh. Math.-Phys. Cl. Königl. Bayer. Akad. Wiss. 16: 146, 151. 1887. Nom. Superfl.

= Serjania hebecarpa fo. amblycephala Radlk., Abh. Math.-Phys. Cl. Königl. Bayer. Akad. Wiss. 16: 146, 151. 1887. Tipo: Paraguay. Paraguari, dans les fôrets, tiges grimpantes, fleurs blanches, 24-IV-1875, B. Balansa 2487a (Lectotipo aquí designado GOET 010850 [foto!]; isolectotipo GOET 010851 [foto!]).

= Serjania hebecarpa fo. platycephala Radlk., Abh. Math.-Phys. Cl. Königl. Bayer. Akad. Wiss. 16: 38, 146, 151. 1887. Tipo: [Argentina. Entre Ríos]. Am Ufer des Uruguay bei Concepcion, IV1875, P. G. Lorentz 170 (Lectotipo aquí designado GOET 010852 [foto!]).

Radlkofer (1887) señala como material tipo de S. hebecarpa Benth. fo. amblycephala el espécimen Balansa 2487a depositado en el herbario Grisebach, que se halla en GOET (GOET 010850 y GOET 010851), es el único citado por Radlkofer en el protólogo de la fo. amblycephala. De estos dos duplicados depositados en GOET, se elige como lectotipo el ejemplar fructífero por definir la forma el ápice del fruto, el isolectotipo es una rama florífera, ambos están determinados por Radlkofer de puño y letra.

De los cinco sintipos citados por Radlkofer (1887) en el protólogo para S. hebecarpa fo. platycephala, se elige como lectotipo el ejemplar Lorentz 170 depositado en GOET (GOET 010852), el que consta de una rama fructífera en excelente estado y está determinado con letra de Radlkofer. 
Serjania incana Radlk., Bull. Herb. Boissier, sér. 2, 3: 805. 1903. = Serjania incana fo. genuina Radlk., Repert. Spec. Nov. Regni Veg. 8: 72. 1910. = Housssayanthus incanus (Radlk.) Ferrucci, Candollea 41: 218. 1986. Tipo: Paraguay. Prope Concepcion in dumeto in arenosis salsis, frutex scandens 10-15, flos albavirens, IX-1901, E. Hassler 7445 (Lectotipo [primer paso], G, designado por M. S. Ferrucci, Candollea 41(1): 218. 1986; lectotipo [segundo paso], aquí designado G 00102861! 2 cartulinas; isolectotipos BM 000087786!, G 00102860 !, G 00102862! 2 cartulinas, $\mathrm{K}$ 000586276!, M 0212303!, S S R-11006!, UC 941279!).

= Serjania humifusa Radlk., Repert. Spec. Nov. Regni Veg. 7: 355. 1909. Tipo: Paraguay. Alto Paraguay, am Boden kriechend im Palmar bei Medanos altitude 100 meters, III-1907, T. C. J. Herzog 589 (Holotipo M 0212309!).

= Serjania incana fo. glabriuscula Radlk., Repert. Spec. Nov. Regni Veg. 8: 72. 1910. Tipo: Paraguay. Chaco septentrionalis: Puerto Talavera in "palmares", scandens $2 \mathrm{~m}$, petala alba, IX1907, K. Fiebrig 1287 (Lectotipo [primer paso], G, designado por M. S. Ferrucci, Candollea 41(1): 218. 1986; lectotipo [segundo paso], aquí designado G 00102863!; isolectotipos G 00102864 ! 2 cartulinas, M 0212301 !, S S-R-11007!).

Radlkofer (1903) en el protólogo de Serjania incana detalla lugar de colección, fecha, colector y número, pero no indica el herbario donde está depositado el espécimen. Se hallaron ocho duplicados de la colección Hassler 7445. Ferrucci (1986) menciona el ejemplar Hassler 7445 depositado en $\mathrm{G}$ como holotipo. Esto se toma como una lectotipificación inadvertida (Prado et al., 2015). Como en $G$ hay dos duplicados, en este trabajo se procede a una lectotipificacion en segundo paso. El ejemplar seleccionado presenta dos ramas floríferas muy bien conservadas y está identificado de puño y letra por Radlkofer, consta de dos cartulinas, en la segunda solo está la descripción impresa de la publicación original.

En el protólogo de Serjania humifusa, Radlkofer (1909) menciona la colección de Herzog 589 consignando la localidad “... im Palmar bei Medanos (Alto Paraguay)...”. El herbario de Herzog depositado originalmente en JE (Stafleu \& Cowan, 1976-1988), fue posteriormente vendido a
L. El único ejemplar localizado de Herzog, cuyos datos de colección coinciden con el protólogo está depositado en M, se trata del holotipo, identificado de puño y letra por Raldkofer (VIII-09); consta de dos ramas, una de ellas con escasas flores. Este espécimen está documentado en la colección de negativos fotográficos del Field Museo bajo $\mathrm{F}$ N-005969.

Radlkofer (1910) en el protólogo de Serjania incana fo. glabriuscula cita el espécimen Fiebrig no. 1287 depositado en el herbario Hassler. Ferrucci (1986) mencionó al ejemplar Fiebrig 1287 depositado en $\mathrm{G}$ como holotipo, pero sería una lectotipificación inadvertida (Prado et al., 2015). Sin embargo, como en G hay dos ejemplares, aquí se procede a una lectotipificación en segundo paso de la especie. Se elige el material G 00102863 como lectotipo, el mismo consta de dos ramas floríferas y posee una etiqueta de puño y letra de Radlkofer donde diferencia las dos formas, la típica y ésta, e indica el material estudiado, la misma está fechada en noviembre de 1909. El otro espécimen depositado en $\mathrm{G}$ ( $\mathrm{G}$ 00102864) no estuvo disponible para Raldkofer, ya que fue adquirido e insertado en la colección general del Herbier Delessert en 1911; este ejemplar consta de una rama florífera con un fruto casi maduro. A su vez se encontraron duplicados de la colección original en $\mathrm{M}$ y S.

Serjania longistipula Radlk., Bot. Jahrb. Syst. 37(1): 148. 1905. Tipo: Peru [Perú]. S. d., A. Weberbauer 537 (Lectotipo aquí designado $\mathrm{F}$ N-005561 [foto!]).

Radlkofer (1905) en el protólogo de Serjania longistipula menciona dos sintipos, ambos depositados en B, donde Weberbauer envió su colección principal, además de los duplicados distribuidos a instituciones americanas y otras europeas, incluido el herbario del Museo de Historia Natural (WRSL) de su ciudad natal, Breslavia (Wroclaw), actualmente situado en Polonia. La mayoría de sus colecciones en Berlín fueron destruidas durante la Segunda Guerra Mundial. Estos números, 518 y 537, de colección de Weberbauer no se han encontrado en ningún herbario peruano ni en ningún otro herbario con la información disponible, de modo que se elige como lectotipo el espécimen Weberbauer 537 que estuviere depositado en $\mathrm{B}$, foto $\mathrm{F} \mathrm{N}-005561$ que 
ilustra una rama florífera, material identificado con letra de Radlkofer y fechado por él en febrero de 1905.

Serjania marginata Casar., Nov. Stirp. Bras. 5: 44. 1843. Tipo: Brazil. S.d. [before 1840], Casaretto Herb. No. 2728 (Clausen s.n.) (Lectotipo TO [2 cartulinas], designado por P. G. Delprete et al., Taxon 68(4): 783-827. 2019; isolectotipos, F No. 695092 [fragment ex G], G 00446570, K 000037184).

= Serjania marginata Casar. fo. pluridentata Radlk., Monogr. Serjania 160. 1875. Tipo: Brésil [Brasil]. Rio de Janeiro, Serra de Jacarepagua, liane, fl. blanche, 22-VII-1867, A. F. M. Glaziou 1023 (Lectotipo aquí designado BR 0000005858163 [foto!]; isolectotipos BR 0000005857142 [foto!], BR 0000005858163 [foto!], M 0212453 [foto!], P 05242920 [foto!], R 000008246 !]).

= Serjania marginata Casar. fo. isopterygia Radlk., Monogr. Serjania 160. 1875. Tipo: Brasilia [Brasil]. S. d., F. Sellow s.n. (Lectotipo aquí designado M 0212451 [foto!]; isolectotipo M 0212452 [foto!]). Syn. nov.

De los dos sintipos citados por Radlkofer (1875) en el protólogo de Serjania marginata fo. pluridentata, se eligió la colección de Glaziou 1023 por estar muy bien preservada y mejor distribuida en los herbarios. Del material accesible, los dos sintipos depositados en BR están identificados de puño y letra por Radlkofer, sin embargo el ejemplar BR 0000005858163 es el seleccionado como lectotipo por responder a un excelente dibujo (M 0212455, foto!), ilustración a lápiz que fue indicada como ilustración del material original de esta forma (Bräuchler, C., 2014/04/08, cita extraída de JSTOR). El ejemplar depositado en $\mathrm{P}$ es el único que tiene una etiqueta manuscrita a lápiz con los datos completos del ejemplar.

De los dos sintipos citados por Radlkofer (1875) en la descripción original de Serjania marginata fo. isopterygia, en las bases de datos consultadas se encontraron dos duplicados del ejemplar Sellow n. 1818, ambos depositados en $\mathrm{M}$, se selecciona como lectotipo el espécimen $\mathrm{M}$ 0212451 por su estado de conservación y por ser una rama más representativa. Estos especímenes fueron enviados por el herbario B. Los caracteres foliares y las flores de mayor tamaño indicados por
Radlkofer para reconocer esta forma son variables en la especie, por lo cual se reconoce solo el nivel específico.

Serjania monogyna Hoffmanns. ex Schltdl., Linnaea 18: 55. 1844. Tipo: Brasilia [Brasil]. Pará. 1801-1807, F. W. Sieber pro J. C. von Hoffmannsegg s.n. (Lectotipo HAL 0090517!; isolectotipos B B-W 07722-010!], BR 0000005415915!, F N-005610 [foto]!).

= Paullinia monogyna (Hoffmanns. ex Schltdl.) Radlk., Monogr. Serjania 74. 1875.

= Paullinia monogyna (Hoffmanns. ex Schltdl.) Radlk. fo. major Radlk., Abh. Math.-Phys. Cl. Königl. Bayer. Akad. Wiss. 19: 300. 1896. Nom. Superfl.

= Paullinia monogyna fo. minor Radlk., Monogr. Paullinia 300. 1895a. Tipo: Brasil. Rondônia. Falls of Madeira, X-1886, H. H. Rusby 539 (Lectotipo aquí designado NY 01139065 [foto!]; isolectotipos NY 01139066!, MICH 1565967!).

Schlechtendal (1844) en el protólogo de Serjania monogyna indica lugar de colección y colector, (Sieber), pero no explicita el herbario donde está depositado el ejemplar. Se localizaron tres duplicados, de la colección original, y una foto $\mathrm{F}$ $\mathrm{N}-005610$ que corresponde al espécimen depositado en B. El ejemplar aquí seleccionado como lectotipo es el depositado en HAL, lugar donde trabajaba Schlechtendal, presenta una rama florífera muy bien conservada y está identificado con letra del autor bajo Seriana monogyna. El espécimen depositado en B (B-W 07722-010) es el más completo, debido a que además de flores presenta frutos inmaduros, sin embargo Schlechtendal no estudió este espécimen porque al finalizar la descripción de la especie indica que no dispuso de material con fruto.

Radlkofer (1895a) en el protólogo de Paullinia monogyna fo. minor menciona dos sintipos, si bien se han localizado ambas colecciones, se designa como lectotipo el espécimen Rusby 539 depositado en NY (NY 01139065) por estar mejor representado. El mismo consta de una rama florífera y presenta una etiqueta manuscrita por Raldkofer con los siguientes datos "Paullinia monogyna m. in Serjania Monogr. 1875, p. 74, n. 97, forma 2 minor. VII.93". El segundo ejemplar depositado en NY (NY 01139066) no posee una etiqueta con la identificación de Radlkofer pero es material original. 
Thinouia mucronata Radlk., Sitzungsber. Math.Phys. Cl. Königl. Bayer. Akad. Wiss. München 8: 282. 1878. Tipo: Brazil, São Paulo, 1875, H. Mosén 3953 (Lectotipo S 17-1112, designado por A. A. Massing \& S. T. Sfoggia Miotto, Phytotaxa 428(2): 159. 2020; isolectotipo S 17-1101).

= Thinouia repanda Radlk., en Engler \& Prantl, Nat. Pflanzenfam. 3(5): 308. 1895b. Tipo: Paraguay. Yaguaron, ciges grimpantes, II-1877, B. Balansa 2488 (Lectotipo aquí designado G 00008258 [foto! 2 cartulinas]; isolectotipos K 000634087!, P 04795732 [foto!]).

En el protólogo de Thinouia repanda Radlkofer (1895b) menciona solo el ejemplar Balansa 2488. Conforme a Stafleu \& Cowan (1976-1988) se desconoce donde está depositado el herbario de Balansa, solo destaca que en $\mathrm{P}$ se halla un set importante. De los tres duplicados localizados en G, P y K, se ha elegido como lectotipo el depositado en G (G 00008258) por ser el único determinado con letra de Radlkofer; el mismo consta de una rama fructífera muy bien preservada.

\section{Agradecimientos}

Agradezco a los curadores de los herbarios consultados por el envío de información y/o imágenes digitales que contribuyeron para este trabajo (B, BA, BM, CORD, E, G, GH, GOET, H, JE, K, LE, M, MICH, MO, PR, SI, TO, WRSL, Z); y a los de los herbarios visitados oportunamente. A Pedro Acevedo-Rodríguez por atender mis consultas y a los revisores anónimos por sus comentarios que contribuyeron a mejorar el manuscrito. Este trabajo ha sido subsidiado por la Agencia Nacional de Promoción Científica y Tecnológica, por el Consejo Nacional de Investigaciones Científicas y Técnicas, por la Secretaría General de Ciencia y Técnica de la Universidad Nacional del Nordeste y por becas de la Myndel Botanica Foundation.

\section{Biblografía}

ACEVEDO-RODRÍGUEZ, P. 2012. 127. Sapindaceae. En: MOTA DE OLIVEIRA, S. (ed.), Flora of the Guianas. Series A: Phanerogams Fascicle 29. Royal Botanical Garden, Kew, pp. 1-198.
ACEVEDO-RODRÍGUEZ, P., K. J. WURDACK, M. S. FERRUCCI, G. JOHNSON, P. PEDRO DIAS, R. L. G. COELHO, G. V. SOMNER, V. W. STEINMANN, E. A. ZIMMER \& M. T. STRONG. 2017. Generic relationships and classification of tribe Paullinieae (Sapindaceae) with a new concept of supertribe Paulliniodae. Syst. Bot. 42: 96-114. https://doi. org/10.1600/036364417X694926

BENTHAM, G. 1851. Second report on Mr. Spruce's collections of dried plants from North Brazil. Hooker's Journal of Botany and Kew Garden Miscellany, vol. 3, pp. 191-200. Reeve and Benham, Henrietta Street, Covent Garden, London.

CAMBÈSSEDES, J. 1828. Sapindaceae. En: SAINTHILAIRE, A. (ed.), Flora Brasiliae Meridionalis (quarto ed.), vol. 1(10), pp. 348-395. A. Belin, Bibliopolam, Via Dicta des Mathurins S.-J. N ${ }^{\circ} 14$, Parisiis.

CAMBÈSSEDES, J. 1834. Note sur deux genres nouveaux de la famille des Sapindacées. Nouv. Ann. Mus. Hist. Nat. 3: 231-238.

DE CANDOLLE, A. P. 1822. Sur les plantes rares ou nouvelles qui ont fleuri dans le Jardin de Botanique de Genève pendant les années 1819,1820 et 1821. Mém. Soc. Phys. Genève 1: 431-463.

DE CANDOLLE, A. P. 1824. Sapindaceae. En: Prodromus systematis naturalis regni vegetabilis, vol. 1, pp. 601618. Treuttel \& Würtz, Paris.

FERRUCCI, M. S. 1986. Nueva combinación en Houssayanthus (Sapindaceae). Candollea 41: 218.

FERRUCCI, M. S. 1987. Houssayanthus monogynus, nueva combinación en Sapindaceae. Candollea 42: 805-807.

FERRUCCI, M. S. 1999. Sapindaceae. En: ZULOAGA, F. O. \& O. MORRONE (eds.), Catálogo de las Plantas Vasculares de la República Argentina II. Monogr. Syst. Bot. Missouri Bot. Gard. 74: 1019-1027.

FERRUCCI, M. S. 2008. Sapindaceae. En: ZULOAGA, F. O., O. MORRONE \& M. J. BELGRANO (eds.), Catálogo de las Plantas Vasculares del Cono Sur (Argentina, sur de Brasil, Chile, Paraguay y Uruguay). Monogr. Syst. Bot. Missouri Bot. Gard. 107: 2938-2951.

GRISEBACH, A. H. R. 1874. Plantae Lorentzianae. Bearbeitung der ersten und zweiten Sammlung argentinischer Pflanzen des Professor Lorentz zu Cordoba. Abh. Königl. Ges. Wiss. Göttingen 19: 49-279.

GRISEBACH, A. H. R. 1879. Symbolae ad floram argentinam. Zweite Bearbeitung argentinischer Pflanzen. Abh. Königl. Ges.Wiss. Göttingen 24: 1-345. https://doi.org/10.5962/bhl.title.9025 
HOOKER, W. J. \& G. A. W. ARNOTT. 1833. En: HOOKER, W. J. (ed.), Contributions towards a flora of South America and the islands of the Pacific, Botanical Miscellany, vol. 3, pp. 129-390. John Murray, Albemarle-street, London.

KERR, J. G. 1894. The botany of the Pilcomayo Expedition; being a list of plants collected during the Argentine expedition of 1890-91 to the Rio Pilcomayo (identifications and the description by Mr. N.E. Brown, Assistant in the Herbarium, Royal Gardens, Kew. Trans \& Proc. Bot. Soc. Edinburgh 20: 44-78.

KUNTH, K. S. 1821. Sapindaceae. En: HUMBOLDT F. W. H. A. V., BONPLAND, A. J. A. \& C. S. KUNTH (eds.), Nova Genera et Species Plantarum (quarto ed.), vol. 5, pp. 77-104. N. Maze, Paris.

MCNEILL, J. 2014. Holotype specimens and type citations: General issues. Taxon 63: 112-113. https://dx.doi.org/10.12705/635.7

PRADO, J. F., R. Y. HIRAI \& R. C. MORAN. 2015. (046-048) Proposals concerning inadvertent lectotypifications (and neotypifications). Taxon 64: 651. https://dx.doi.org/10.12705/643.29

RADLKOFER, L. 1875. Monographie der SapindaceenGattung Serjania. Verlag der K. B. Akademie, München.

RADLKOFER, L. 1878. Ueber Sapindus und damit in Zusammenhang stehende Pflanzen. Sitzungsber. Math.-Phys. Cl. Königl. Bayer. Akad. Wiss. München 8: 221-408.

RADLKOFER, L. 1887. Ergänzungen zur Monographie der Sapinaceen-Gattung Serjania. Abh. Math.-Phys. Cl. Königl. Bayer. Akad. Wiss. 16: 38, 146, 151.

RADLKOFER, L. 1895a. Monographie der SapindaceenGattung Paullinia. Abh. Math.-Phys. Cl. Königl. Bayer. Akad. Wiss. 19(1): 67-381.

RADLKOFER, L. 1895b. Sapindaceae. En: ENGLER, A. \& K. PRANTL, Die natürlichen Pflanzenfamilien, III, vol. 5, pp. 277-366. Verlag von Wilhelm Engelmann, Leipzig.

RALKOFER, L. 1896. An enumeration of the plants collected in Bolivia by Miguel Bang, with description of new genera and species. Part 3. Mem. Torrey Bot. Club 6: 1-130.

RADLKOFER, L. 1892-1900. Sapindaceae. En: MARTIUS, C. F. P., Flora Brasiliensis, vol. 13(3), pp. 225-658, tab. 58-123. R. Oldenbourg in comm., Monachii et Lipsiae.

RADLKOFER, L. 1903. Sapindaceae. En: CHODAT R. \& E. HASSLER, Plantae Hasslerianae. Bull. Herb. Boissier. II, 3: 803-809.
RADLKOFER, L. 1905. Sapindaceae novae e generibus Serjania et Paullinia. Bot. Jahrb. Syst. 37: 144-155.

RADLKOFER, L. 1909. Sapindaceae. En: HERZOG, TH. VON, Nachträge zu Siphonogamae novae Bolivienses. Repert. Spec. Nov. Regni Veg. 7: 354356. https://doi.org/10.1002/fedr.19090072208

RADLKOFER, L. 1910. Ex herbario Hassleriano: novitates Paraguariensis V. Repert Spec. Nov. Regni Veg. 8: 71-73.

https://doi.org/10.1002/fedr.4870081014

RADLKOFER, L. 1913. Sapindaceae. En: Die von Dr. Th. Herzog auf seiner zweiten Reise durch Bolivien in den Jahren 1910 und 1911 gesammelten Pflanzen. Meded. Rijks-Herb. 19: 58-63.

RADLKOFER, L. 1931-1934. Sapindaceae. En: ENGLER, H. G. A. (ed.), Das Planzenreich 4, 165 (Heft 98): 1-1539. Verlag von H. R. Engelmann (J. Cramer), Weinheim.

SAINT-HILAIRE, A. 1823. Description des principaux genres nouveaux et des espèces nouvelles de la Flore du Brésil, cités dans le Mémoire sur le Gynobase. Bull. Sci. Soc. Philom. Paris 1823: 129-133.

SCHLECHTENDAL, D. F. L. DE. 1844. De plantis mexicanis a G. Schiede, M. Dr., Car. Ehrenbergio aliisque, colectis nuntium adfert. Linnaea 18: 48-64.

STAFLEU, F A. \& R. S. COWAN. 1976-1988. Taxonomic literature: a selective guide to botanical publications and collections with dates, commentaries and types. $2^{\mathrm{a}}$ ed. Taxonomic literature II. https://doi.org/10.5962/bhl.title.48631

SWARTZ, O. 1788. Nova genera et species plantarum seu Prodromus. Bibliopoliis Acad. M. Swederi, Holmiae, Upsaliae, \& Aboae.

THIERS, B. (continuamente actualizado). 2021. Index Herbariorum: a global directory of public herbaria and associated staff. New York Botanical Garden's virtual herbarium. https:// sweetgum.nybg.org/ science/ih/ [Último acceso: mayo de 2021].

TURLAND, N. J., J. H. WIERSEMA, F. R. BARRIE, W. GREUTER, D. L. HAWKSWORTH, P. S. HERENDEEN, S. KNAPP, W.-H. KUSBER, D.-Z. LI, K. MARHOLD, T. W. MAY, J. MCNEILL, A. M. MONRO, J. PRADO, M. J. PRICE \& G. F. SMITH. 2018. International Code of Nomenclature for algae, fungi, and plants (Shenzhen Code), Regnum Vegetabile 159. Glashütten: Koeltz Botanical Books. https://doi.org/10.12705/Code.2018

WEST, J. G. 1984. A revisión of Dodonaea Miller (Sapindaceae) in Australia. Brunonia 7: 1-194. https://doi.org/10.1071/BRU9840001 